\title{
Metal concentrations in the upper atmosphere during meteor showers
}

\author{
J. Correira ${ }^{1, *}$, A. C. Aikin ${ }^{1}$, J. M. Grebowsky ${ }^{2}$, and J. P. Burrows ${ }^{3}$ \\ ${ }^{1}$ The Catholic University of America, Institute for Astrophysics and Computational Sciences Department of Physics, \\ Washington, DC 20064, USA \\ ${ }^{2}$ NASA Goddard Space Flight Center, Code 695, Greenbelt, MD 20771, USA \\ ${ }^{3}$ Institute of Environmental Physics (IUP), University of Bremen, Bremen, Germany \\ "now at: Computational Physics, Inc., Springfield, VA 22151, USA
}

Received: 30 June 2009 - Published in Atmos. Chem. Phys. Discuss.: 10 September 2009

Revised: 4 January 2010 - Accepted: 14 January 2010 - Published: 1 February 2010

\begin{abstract}
Using the nadir-viewing Global Ozone Measuring Experiment (GOME) UV/VIS spectrometer on the ERS2 satellite, we investigate short term variations in the vertical magnesium column densities in the atmosphere and any connection to possible enhanced mass deposition during a meteor shower. Time-dependent mass influx rates are derived for all the major meteor showers using published estimates of mass density and temporal profiles of meteor showers. An average daily sporadic background mass flux rate is also calculated and used as a baseline against which calculated shower mass flux rates are compared. These theoretical mass flux rates are then compared with GOME derived metal vertical column densities of $\mathrm{Mg}$ and $\mathrm{Mg}^{+}$from the years 19962001. There is no correlation between theoretical mass flux rates and changes in the $\mathrm{Mg}$ and $\mathrm{Mg}^{+}$metal column densities. A possible explanation for the lack of a shower related increase in metal concentrations may be differences in the mass regimes dominating the average background mass flux and shower mass flux.
\end{abstract}

\section{Introduction}

Meteor showers are the most obvious manifestation of the interaction between meteors and the Earth's atmosphere. Written records going back thousands of years describe in vivid terms what we today call colloquially "shooting stars" raining down from the heavens. Apart from the impressive visual

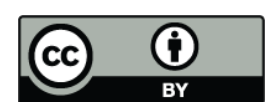

Correspondence to: J. Correira (john.correira@gmail.com) display, the impact of meteor showers on the Earth's atmosphere has been a matter of debate. Various sources assert that meteor showers are a small fraction of the total mass input to Earth (Williams, 2001), while other authors state that there could be observable effects of meteor showers in the total abundance of meteoric atoms in the atmosphere (e.g. McNeil et al., 2001). Studies have investigated the E-region of the ionosphere during meteor showers and found enhancements during showers (e.g. Zhou et al., 1999), but these seem to be of a transient nature.

In addition to being an awe inspiring sight, meteors are also of interest because they allow the general public to make a significant contribution to scientific research, much as amateur astronomers do with the magnitudes of variable stars. This paper and similar analysis would be impossible without the contribution of these observers. The work of Jenniskens (1994), used extensively in this work, relies on the data collected by 16 observers, totaling $4482 \mathrm{~h}$ of observations over the span of several years. The detection of visual meteors can also be accomplished by way of video observation (Hawkes and Jones, 1986; Hawkes, 1993), with several sophisticated software packages available for post-detection analysis (Molau and Gural, 2005). The availibility of CCD's and their relatively inexpensive cost has encouraged the continue growth of this method of observation.

Here we will utilize GOME (Global Ozone Monitoring Experiment) data, from the ERS-2 satellite, to probe short term variations in the magnesium atoms (neutrals and ions) vertical column densities and explore the connection to the increased deposition of mass during a meteor shower. The ERS-2 satellite was launched on 21 April 1995. The spacecraft is in a retrograde, sun-synchronous, $795 \mathrm{~km}$

Published by Copernicus Publications on behalf of the European Geosciences Union. 
high near-polar orbit with an equatorial crossing time of 10:30 a.m. LT (local time) and an orbital period of about $100 \mathrm{~min}$. GOME data is available from 1996 until June 2003, when an on-board tape recorder failed. It measured earthshine spectra for wavelengths from 237 to $793 \mathrm{~nm}$. The GOME instrument was designed to focus on the distributions of atmospheric constituents such as ozone, nitrogen dioxide, formaldehyde, bromine oxide, and water vapor but its spectral range includes lines of several metal atom species and their ions.

\section{Satellite data analysis}

The determination of metal column densities using the GOME instrument was first described in Aikin et al. (2005). The vertical column densities of metal atoms are derived from GOME photon counts using the airglow equation

$4 \pi J_{m}=g \int n(z) d z$

where $J_{m}$ is the radiance integrated over a metal spectral line, $\int n d z$ is the sought after vertical column density, and $g$ is the column photon emission rate, the " $g$-factor", which is given by Paxton and Anderson (1992)

$g=\frac{\pi e^{2}}{m c^{2}} \lambda^{2} f \pi F\left(\lambda_{0}\right) P(\theta)$

with $\pi F\left(\lambda_{0}\right)$ the solar irradiance at the transition wavelength in units of photons per $\mathrm{cm}^{2}$ per second per nanometer and $f$ is the transition oscillator strength. We have included the anisotropic scattering phase function $P(\theta)$ (see Chandrasekhar, 1960, p.50), where $\theta$ is the angle between the incident solar photon and the scattered photon. The symbols $\lambda$, $e, m$, and $c$ represent wavelength, electron charge, electron mass and the speed of light, respectively. Rotational Raman Scattering (RRS), also known as the Ring Effect, is the inelastic scatter of solar photons and fills in the metal emission lines. We fully corrected the derived metal column densities for RRS for by using the SCIATRAN radiative transfer model (Rozanov et al., 2005) to estimate the magnitude of the filling-in as a function of solar zenith angle. Using this analysis Correira et al. (2008) studied the seasonal variations of the metal column densities. In the present paper the effects of the fleeting deposition of meteor showers will be explored.

\section{Mass input from meteor showers}

Jenniskens (1994) found that the temporal variation of the Zenithal Hourly Rates (ZHR), the number of visible meteors an observer could see under ideal conditions, of most meteor showers could be described by

$\operatorname{ZHR}\left(\lambda_{\odot}\right)=\mathrm{ZHR}_{\max } 10^{-B\left|\lambda_{\odot}-\lambda_{\odot}^{\max }\right|}$ where $\lambda \odot$ is the solar (or ecliptic) longitude, $\lambda_{\odot}^{\max }$ and $Z_{\text {ZHR }}$ max are the solar longitude and ZHR at the peak of the shower, respectively, and $B$ is the slope of a line fitted to the ZHR activity profiles when plotted on a logarithmic scale. If it is assumed that the mass density of the stream is proportional to the ZHR, then from Eq. (3) we can write in a similar way

$\rho\left(\lambda_{\odot}\right)=\rho_{\max } 10^{-B\left|\lambda_{\odot}-\lambda_{\odot}^{\max }\right|}$

with $\rho\left(\lambda_{\odot}\right)$ the mass density of a stream at solar longitude $\lambda_{\odot}$ and $\rho_{\max }$ is the peak mass density of the stream. The mass accumulation rate onto the Earth, $\Phi$, can be estimated by

$\Phi=\rho V_{G}\left(\pi R_{\oplus}^{2}\right)$

where $V_{G}$ is the geocentric velocity of the meteor stream and $R_{\oplus}$ is the Earth's radius. Inserting Eq. (4) into Eq. (5) yields a time-dependent expression for the incident mass flux into the Earth's atmosphere

$\Phi=\rho_{\max } V_{G}\left(\pi R_{\oplus}^{2}\right) 10^{-B\left|\lambda_{\odot}-\lambda_{\odot}^{\max }\right|}$.

The total mass accumulated by Earth due to a meteor stream, $M_{T}$ can then be found by integrating over the duration of the shower:

$M_{T}=\int_{\lambda_{\odot}^{\text {start }}}^{\lambda_{\odot}^{\text {end }}} \Phi d \lambda_{\odot}$.

Another method to calculate the total mass accumulated by the Earth is to use the "equivalent duration" (Hughes and McBride, 1989) or "equivalent cross-section" (Jenniskens, 1994), $\Delta t$, of the meteor shower. Multiplying the equivalent cross-section by the peak mass flux gives the total mass swept up by the Earth during its passage through the stream. The equivalent cross-section can be calculated from

$\Delta t=\int 10^{-B\left|\lambda_{\odot}-\lambda_{\odot}^{\max }\right|} d \lambda_{\odot}$.

If the slope of the ZHR activity is given by

$B=\left\{\begin{array}{l}B_{+} \text {if } \lambda_{\odot}<\lambda_{\odot}^{\max } \\ B_{-} \text {if } \lambda_{\odot}>\lambda \lambda_{\odot}^{\max }\end{array}\right.$

then the equivalent cross-section is

$\Delta t=\frac{1}{\ln (10)}\left(\frac{1}{B_{+}}+\frac{1}{B_{-}}\right)$.

For most showers the rise and decay of the ZHR profile is symmetrical, i.e. $B_{+}=B_{-}$, leaving

$\Delta t=\frac{2}{\ln (10)} \frac{1}{B}$.

Jenniskens (1994) found a few meteor streams that present more complex profiles, requiring two sets of exponentials for 


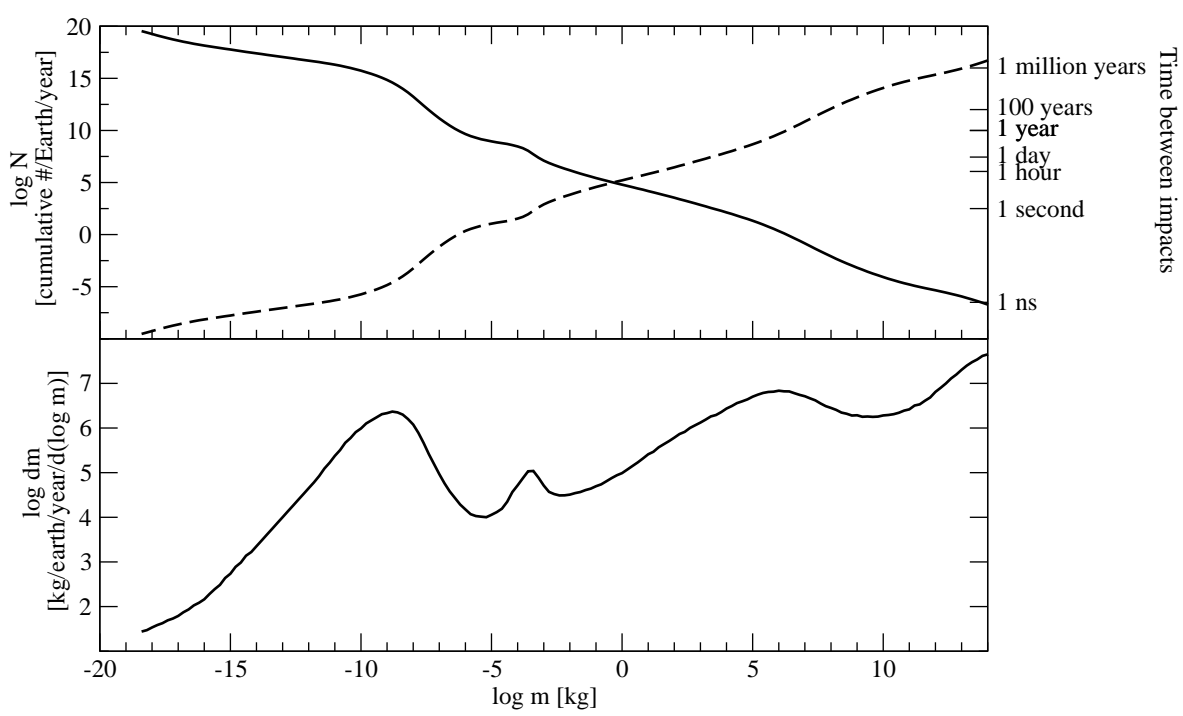

Fig. 1. Upper panel: Solid line represents the cumulative number of meteors with mass $m$ or greater intercepted by the Earth per year as a function of meteor mass. The dashed line represents the time between Earth impacts of particles with mass $m$ or greater, with several important time scales marked on the right axis. Lower panel: increment of mass intercepted by Earth per year per increment of logarithmic mass of incoming meteors. Adapted from Ceplecha et al. (1998).

a proper fit, one set describing the long term activity of the shower, called the background (not to be confused with the constant background flux of sporadic meteors), and the other set of exponentials describing the main peak of the shower. Using $p$ and $b$ for the peak and background components, respectively, Eq. (9) would be replaced by

$\Delta t=\frac{1}{\ln (10)}\left(\frac{1}{B^{p+}}+\frac{1}{B^{p-}}+\frac{1}{B^{b+}}+\frac{1}{B^{b-}}\right)$.

One shower in particular, the Geminids, has an asymmetrical peak requiring all four terms in Eq. (11). For two component (i.e. background + peak) showers with a symmetrical peak, $B^{p+}=B^{p-}=B^{p}$. Where the meteor shower background begins is somewhat ambiguous and so the slope of the background is assumed to be symmetrical and the value for the descending background, $B^{b-}$, is used (Peterson, 1999), leaving

$\Delta t=\frac{2}{\ln (10)}\left(\frac{1}{B^{p}}+\frac{1}{B^{b-}}\right)$

\section{Results}

\subsection{Calculated}

How large an effect meteor showers have on the metal content of the atmosphere depends largely on their mass deposition rate compared to the daily background mass deposition. The mass input due to showers may be insignificant or be a substantial fraction of the daily mass input. In order to investigate the contribution from showers relative to the daily sporadic background influx the mass flux rates from both sources need to be estimated.

Starting with the sporadic background mass flux, we look to the work of Ceplecha et al. (1998) which compiles the results from 6 prior works to derive a per year mass flux rate. The total mass flux across the range $10^{-21}$ to $10^{15} \mathrm{~kg}$ was estimated to be $\sim 1.3 \times 10^{8} \mathrm{~kg}$ per year over the entire surface of the Earth. Figure 1 shows the mass flux rates estimated by Ceplecha et al. (1998). The dashed line and time labels on the right hand side indicate the average incident times between particles of particular masses. Meteors of a very large mass dominate the magnitude of the mass flux rate, yet are relatively rare. A meteor of $\sim 10^{7} \mathrm{~kg}$ will, on average, enter the Earth's atmosphere only once every hundred years, and therefore should not be considered when investigating the average daily background flux in relation to meteor showers. Hence, the upper mass limit of ablating meteors is effectively set by the time scale of interest. Ceplecha et al. (1998) notes that considering the mass influx on the time scale of a human life bodies with masses greater than $10^{8} \mathrm{~kg}$ can be excluded and lowers the average total mass flux by almost an order of magnitude to $2.4 \times 10^{7} \mathrm{~kg}$ per year over the entire surface of the Earth.

Small mass meteors dominate the population swept up the by the Earth every day. Hughes (1997) notes that incoming particles of very small sizes radiate away heat very effectively due to the larger cross sectional area to mass ratio, preventing the particles from reaching their boiling points and undergoing ablation. Hughes (1997) finds a lower ablation limit on the meteor radius of $10^{-3} \mathrm{~cm}$ to $10^{-4} \mathrm{~cm}$ for initial velocities of 20 and $50 \mathrm{~km} / \mathrm{s}$, respectively. Using a common 


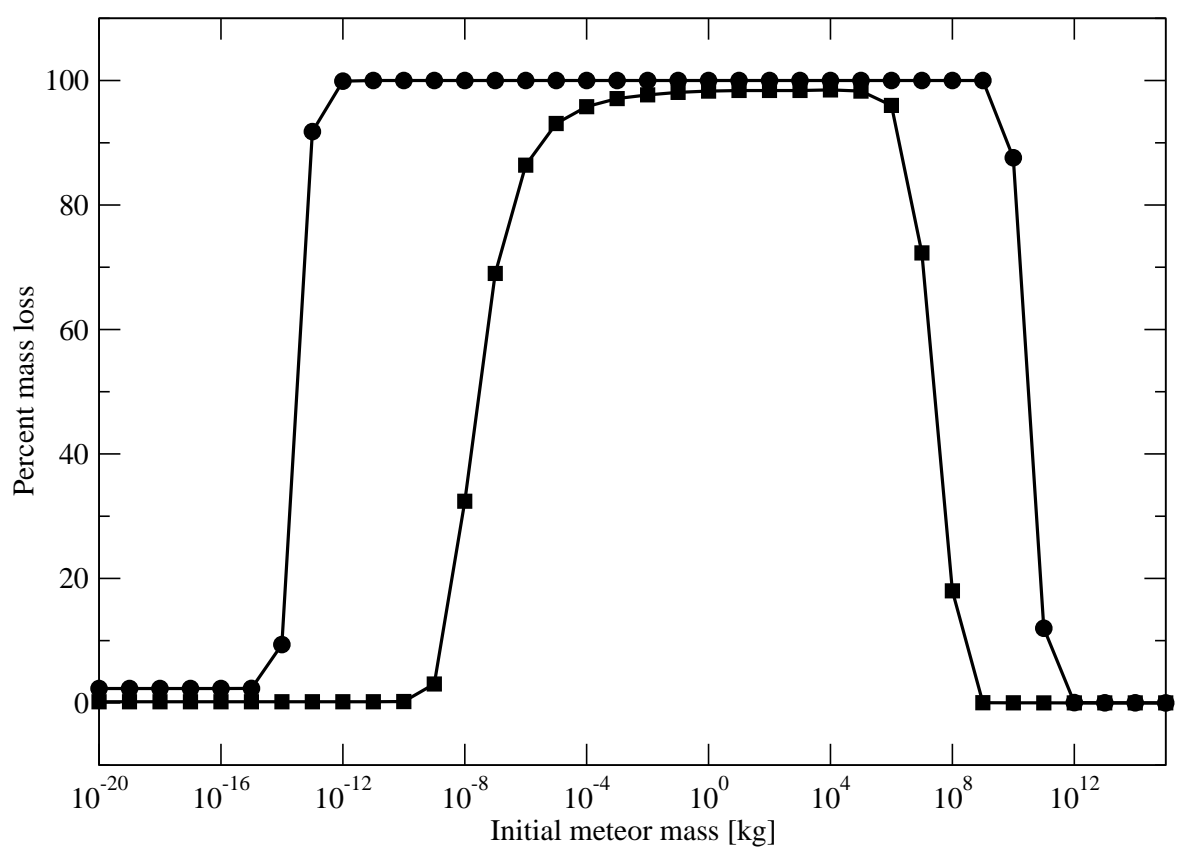

Fig. 2. Percent of initial mass lost due to ablation during passage through the atmosphere as a function of the initial meteor mass. Values are calculated from the ablation code of Pesnell and Grebowsky (2000). Squares are for meteors with an inital velocity of $20 \mathrm{~km} / \mathrm{s}$, circles are for meteors with an initial velocity of $72 \mathrm{~km} / \mathrm{s}$, typical of a high speed shower such as the Leonids.

value of $3 \mathrm{~g} \mathrm{~cm}^{-3}$ (see, e.g. p. 350, Grün et al., 2001) for a stoney meteor particle density and approximating the meteor as a sphere, a particle radius of $10^{-3} \mathrm{~cm}$ corresponds to a mass of about $10^{-11} \mathrm{~kg}$, and a particle radius of $10^{-4} \mathrm{~cm}$ corresponds to a meteor mass of about $10^{-14} \mathrm{~kg}$. Particles smaller than this will not ablate and will simply float downward in the atmosphere under the influence of gravity.

Using the ablation code of Pesnell and Grebowsky (2000), we can model the ablation of meteors over a range of masses and entry velocities to confirm this interpretation. Figure 2 shows the ablated mass as a percentage of the initial meteor mass for two different initial velocities: $20 \mathrm{~km} \mathrm{~s}^{-1}$ (denoted by squares), the nominal speed of the sporadic background; and $72 \mathrm{~km} \mathrm{~s}^{-1}$ (denoted by circles), corresponding to the incident speed of meteors from a high speed shower, such as the Leonids. Both velocities are for an impact angle of $45^{\circ}$. For a large range of masses there is nearly complete ablation. At the high and low mass limits there is relatively little ablation. On the low mass side the small amount of ablation is due to the effect described by Hughes (1997), discussed above. At the high mass limit the small amount of mass lost due via ablation is due to the fact that the meteor is so large that the Earth's atmosphere does very little to slow the meteor, which ends up impacting the Earth's surface before much of it is ablated. As the average speed of a sporadic meteor is $\sim 20 \mathrm{~km} / \mathrm{s}$, based on the work of Hughes (1997) and our modeling analysis here, it seems reasonable to choose a lower limit cutoff mass of about $10^{-11} \mathrm{~kg}$.
As noted above the upper mass limit is somewhat arbitrary depending on the time scales of interest, but given a range of reasonable time scales, the exact value used for the upper mass limit does not greatly affect the final mass flux rates. Using an upper limit of particles likely to enter the atmosphere during a one year time frame sets an upper mass limit of $\sim 2 \times 10^{6} \mathrm{~kg}$ and, combined with the lower mass limit of $10^{-11} \mathrm{~kg}$, yields a mass flux of $1.7 \times 10^{7} \mathrm{~kg}$ per year over the surface of the Earth; a one week time frame sets an upper mass limit of $\sim 3 \times 10^{4} \mathrm{~kg}$ and a mass flux of $8.8 \times 10^{6} \mathrm{~kg}$ per year over the surface of the Earth; finally, a time frame of one day sets an upper mass limit of $\sim 2.5 \times 10^{3} \mathrm{~kg}$, yielding a mass flux of $5.8 \times 10^{6} \mathrm{~kg}$ per year over the surface of the Earth. A once per year passage of a large meteor through the atmosphere is not likely to create long lasting global effects, therefore an upper mass limit of $3 \times 10^{4} \mathrm{~kg}$ and a mass flux of $8.8 \times 10^{6} \mathrm{~kg}$ per year over the surface of the Earth for the background mass flux is adopted.

Having estimated the relevant background (i.e. sporadic population) mass flux we now turn to the mass deposited on Earth due to meteor streams. To ensure that only clearly identified streams with a fairly consistent record were included, the list of meteor streams from Jenniskens (1994) was cross referenced with the current working list of visual meteor showers compiled by the International Meteor Organization (retrieved from http://www.imo.net/calendar/2006/ tables). The names of some streams have changed over time, but using the solar longitude at the shower peak as well as 
Table 1. Meteors stream properties.

\begin{tabular}{|c|c|c|c|c|c|c|c|c|c|}
\hline Name & Code & start & end & peak & $\lambda$ & R.A. & Dec. & $\mathrm{v}_{\infty}$ & ZHR \\
\hline Quadrantids & QUA & $1 \mathrm{Jan}$ & 5 Jan & 3 Jan & $283^{\circ} 16$ & $230^{\circ}$ & 49 & 41 & 120 \\
\hline$\alpha$ Centaurids & ACE & 28 Jan & $21 \mathrm{Feb}$ & $8 \mathrm{Feb}$ & $319^{\circ} 2$ & $210^{\circ}$ & -59 & 56 & 6 \\
\hline$\delta$ Leonids & DLE & $15 \mathrm{Feb}$ & $10 \mathrm{Mar}$ & $24 \mathrm{Feb}$ & $336^{\circ}$ & $168^{\circ}$ & 16 & 23 & 2 \\
\hline$\gamma$ Normids & GNO & $25 \mathrm{Feb}$ & $22 \mathrm{Mar}$ & 13 Mar & $353^{\circ}$ & $249^{\circ}$ & -51 & 56 & 8 \\
\hline Lyrids & LYR & $16 \mathrm{Apr}$ & $25 \mathrm{Apr}$ & $22 \mathrm{Apr}$ & $32^{\circ} 32$ & $271^{\circ}$ & 34 & 49 & 18 \\
\hline$\eta$ Aquarids & ETA & 19 Apr & 28 May & 6 May & $45^{\circ} 5$ & $338^{\circ}$ & -1 & 66 & 60 \\
\hline July Phoenicids & PHE & $10 \mathrm{Jul}$ & $16 \mathrm{Jul}$ & $13 \mathrm{Jul}$ & $111^{\circ}$ & $32^{\circ}$ & -48 & 47 & var \\
\hline Piscis Austrinids & PAU & $15 \mathrm{Jul}$ & $10 \mathrm{Aug}$ & $28 \mathrm{Jul}$ & $125^{\circ}$ & $341^{\circ}$ & -30 & 35 & 5 \\
\hline South $\delta$ Aquarids & SDA & $12 \mathrm{Jul}$ & 19 Aug & $28 \mathrm{Jul}$ & $125^{\circ}$ & $339^{\circ}$ & -16 & 41 & 20 \\
\hline$\alpha$ Capricornids & CAP & $3 \mathrm{Jul}$ & 15 Aug & $30 \mathrm{Jul}$ & $127^{\circ}$ & $307^{\circ}$ & -10 & 23 & 4 \\
\hline South $\iota$ Aquarids & SIA & $25 \mathrm{Jul}$ & 15 Aug & 4 Aug & $132^{\circ}$ & $334^{\circ}$ & -15 & 34 & 2 \\
\hline North $\delta$ Aquarids & NDA & $15 \mathrm{Jul}$ & 25 Aug & 8 Aug & $136^{\circ}$ & $335^{\circ}$ & -5 & 42 & 4 \\
\hline Perseids & PER & $17 \mathrm{Jul}$ & 24 Aug & 12 Aug & $140^{\circ}$ & $46^{\circ}$ & 58 & 59 & 100 \\
\hline$\kappa$ Cygnids & $\mathrm{KCG}$ & 3 Aug & 25 Aug & 18 Aug & $145^{\circ}$ & $286^{\circ}$ & 59 & 25 & 3 \\
\hline$\alpha$ Aurigids & AUR & 25 Aug & 8 Sep & 1 Sep & $158^{\circ} 6$ & $84^{\circ}$ & 42 & 66 & 10 \\
\hline$\epsilon$ Geminids & EGE & $14 \mathrm{Oct}$ & 27 Oct & $18 \mathrm{Oct}$ & $205^{\circ}$ & $102^{\circ}$ & 27 & 70 & 2 \\
\hline Orionids & ORI & 2 Oct & $7 \mathrm{Nov}$ & $21 \mathrm{Oct}$ & $208^{\circ}$ & $95^{\circ}$ & 16 & 66 & 23 \\
\hline Southern Taurids & STA & $1 \mathrm{Oct}$ & $25 \mathrm{Nov}$ & $5 \mathrm{Nov}$ & $223^{\circ}$ & $52^{\circ}$ & 13 & 27 & 5 \\
\hline Leonids & LEO & 14 Nov & $21 \mathrm{Nov}$ & $19 \mathrm{Nov}$ & $235^{\circ} 27$ & $153^{\circ}$ & 22 & 71 & 100 \\
\hline Dec Phoenicids & PHO & $28 \mathrm{Nov}$ & $9 \mathrm{Dec}$ & $6 \mathrm{Dec}$ & $254^{\circ} 25$ & $18^{\circ}$ & -53 & 18 & var \\
\hline Puppids/Velids & PUP & $1 \mathrm{Dec}$ & $15 \mathrm{Dec}$ & $7 \mathrm{Dec}$ & $255^{\circ}$ & $123^{\circ}$ & -45 & 40 & 10 \\
\hline Monocerotids & MON & 27 Nov & $17 \mathrm{Dec}$ & $9 \mathrm{Dec}$ & $257^{\circ}$ & $100^{\circ}$ & 8 & 42 & 3 \\
\hline$\sigma$ Hydrids & HYD & $3 \mathrm{Dec}$ & $15 \mathrm{Dec}$ & $12 \mathrm{Dec}$ & $260^{\circ}$ & $127^{\circ}$ & 2 & 58 & 2 \\
\hline Geminids & GEM & $7 \mathrm{Dec}$ & $17 \mathrm{Dec}$ & $14 \mathrm{Dec}$ & $262^{\circ} 2$ & $112^{\circ}$ & 33 & 35 & 120 \\
\hline Ursids & URS & $17 \mathrm{Dec}$ & $26 \mathrm{Dec}$ & $22 \mathrm{Dec}$ & $270^{\circ} 7$ & $217^{\circ}$ & 76 & 33 & 10 \\
\hline
\end{tabular}

the right ascension and declination of the radiant yielded 24 meteor streams common to both the IMO working list and Jenniskens (1994) list. The analysis discussed in Section 3 was applied to these streams to obtain their mass flux rates. Table 1 lists the details of the selected streams. Where there was a discrepancy between the IMO working list and Jenniskens (1994) the more current information from the IMO working list is used. The Virginids shower was common to both lists but is not included in the current analysis since its profile did not allow for a reliable fit for the activity parameter $B$. Table 2 shows the results of this analysis, including the estimated mass flux rates, total mass deposited to the Earth, and average mass deposition per day.

In order to gain a better sense of the temporal variation in the mass flux rate throughout the year due to meteor showers, Eq. (6) is used to obtain the mass flux rate as a function of solar longitude. The solid black line in Fig. 3 is the result of applying Eq. (6) to the meteor streams listed in Table 1, while the dashed line is the average sporadic background mass flux of $5 \times 10^{-17} \mathrm{grams} \mathrm{cm}^{-2} \mathrm{~s}^{-1}$. The total estimated mass flux rate is the sum of these two and is shown in red.

\subsection{Satellite observations}

From Fig. 3 it is seen that the best opportunity to observe a distinct increase in the metal column density in the atmosphere is during the Geminids (at $262^{\circ}$ solar longitude) and Quadrantids (at $283^{\circ}$ solar longitude, also known as the the Bootids) showers, due to both the large peak magnitudes of their mass flux, and the relative narrowness of the mass flux peak, providing a short pulse of mass deposition. To look for effects in the atmosphere the method of analysis previously described in Sect. 2 was applied to GOME data from years 1996-2001. Resonance lines at $285.2 \mathrm{~nm}$ and $279.9 \mathrm{~nm}$ were used to determine $\mathrm{Mg}$ and $\mathrm{Mg}^{+}$vertical column densities, respectively (see Correira et al., 2008, for further details). GOME reported dates were converted into Julian dates and then solar longitudes in order to avoid any uncertainties due to oddities in the civil calendar (e.g., 1996 and 2000 were leap years). To see if there was any observable shower enhancement in atmospheric metal column densities, all available data from the 6 years of GOME data available were folded together and ordered by solar longitude in order to improve the signal to noise ratio.

The data was further restricted by latitude to focus on the atmosphere region presumably most affected by the shower, i.e. where the angle of incidence of the stream is normal to 
Table 2. Density and mass of meteor streams.

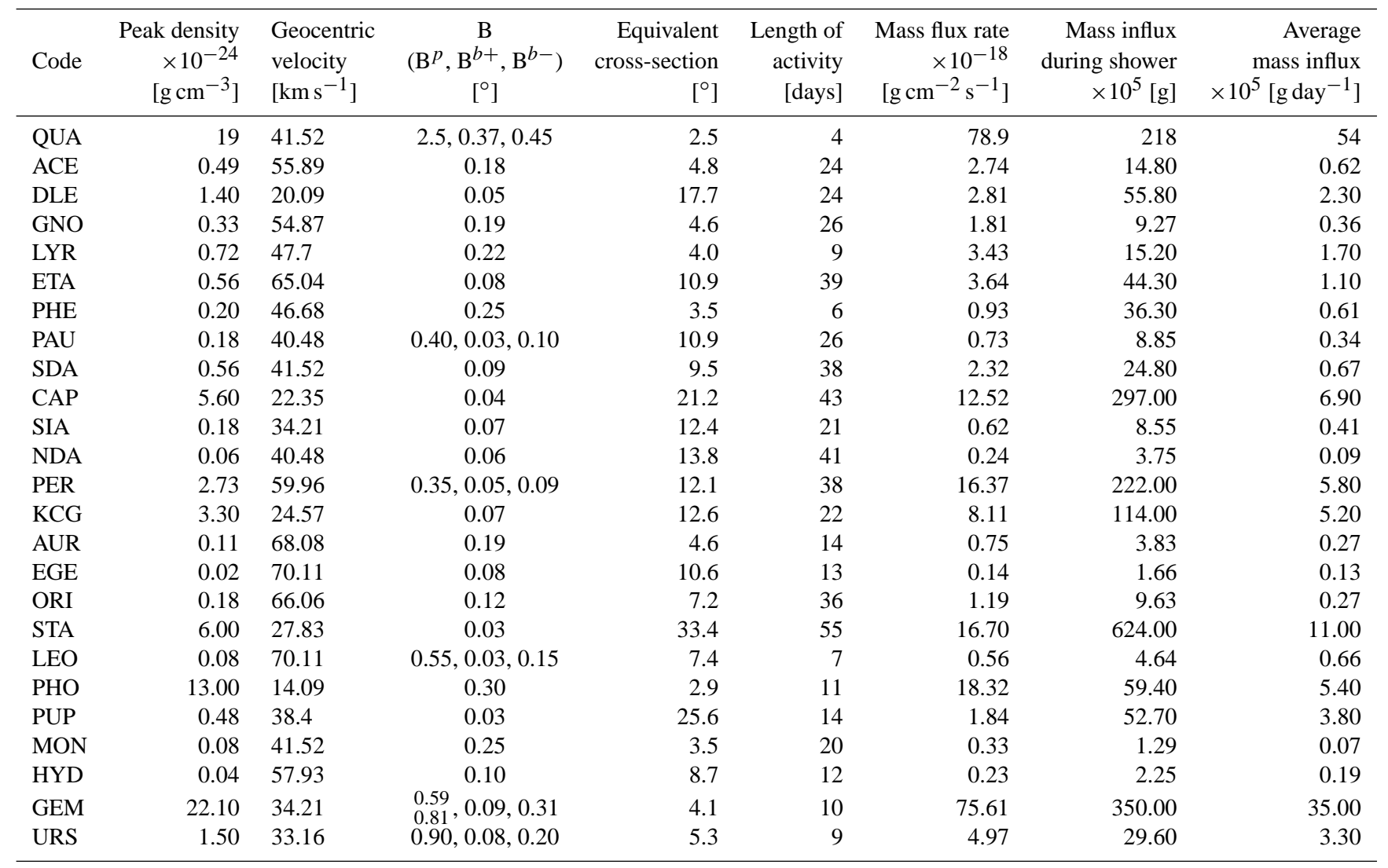

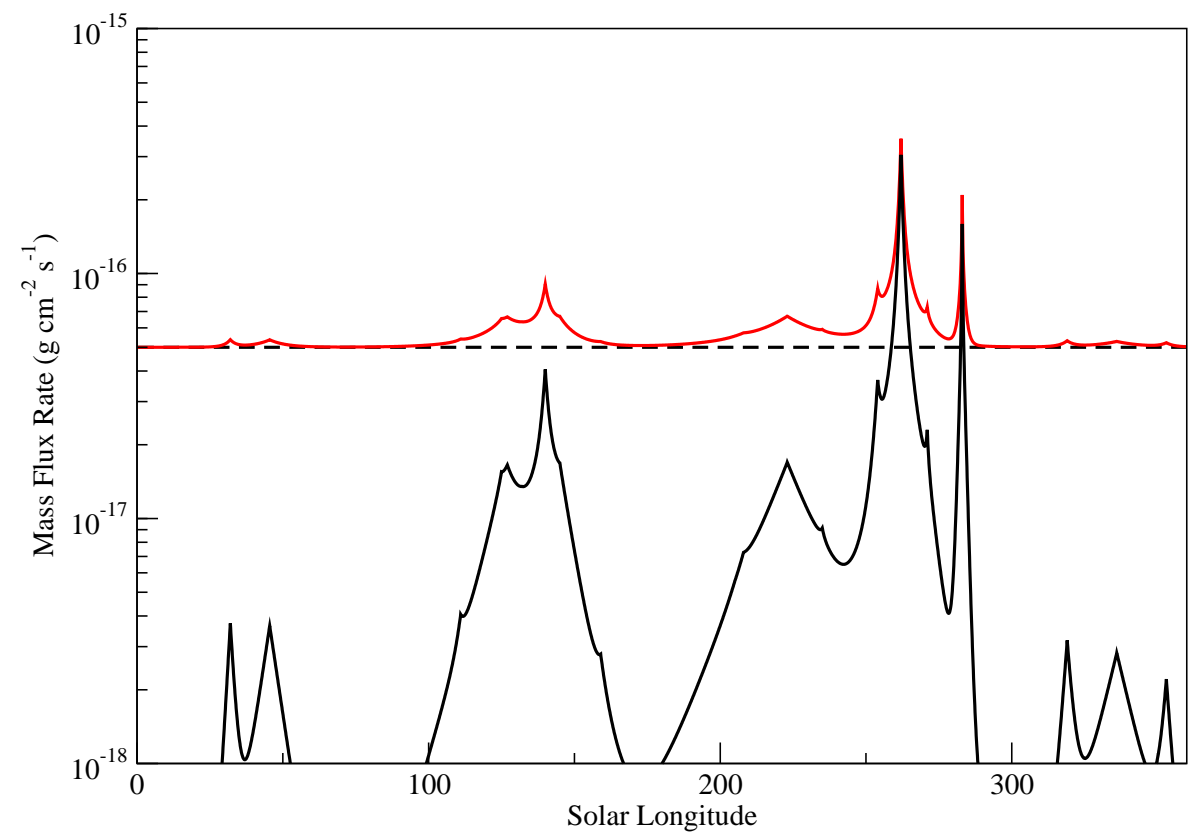

Fig. 3. Solid black line is the mass flux rate as a function of solar longitude for the meteor streams considered in this work. Dashed line is the daily background mass flux of $5 \times 10^{-17}$ grams cm $\mathrm{cm}^{-2} \mathrm{~s}^{-1}$. Note that the peak of several showers coincide or are very close and may appear as only one peak. Red line is the sum of mass flux due to showers and background mass flux. 


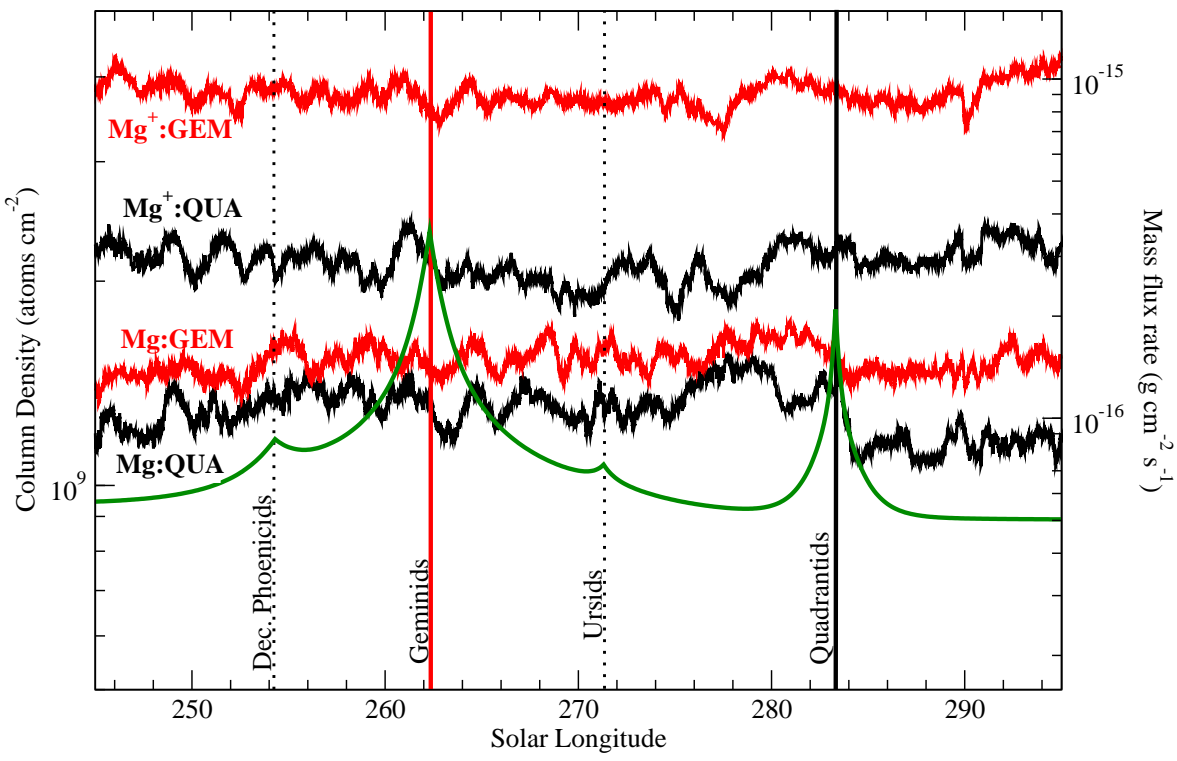

Fig. 4. Column density versus solar longitude. Data were broken into $10^{\circ}$ latitude bands appropriate for the respective shower, $5^{\circ}$ either side of the geodetic latitude corresponding to where the shower radiant is at local zenith. Black represents data for Quadrantids, $44^{\circ}-54^{\circ}$ degrees latitude, while red is for Geminids, $28^{\circ}-38^{\circ}$ degrees north latitude. The thick green line in the estimated total mass flux rate (i.e. sum of mass flux due to showers and background mass flux) and thin vertical black lines are the times of shower peaks.

the Earth's surface. The radiant of a meteor shower will be at the local zenith where the geographic latitude is equal to the radiant's declination. The declination of the Geminids and Quadrantids showers are $+33^{\circ}$ and $+49^{\circ}$ respectively, so GOME data were narrowed to latitude bands of $28^{\circ}-38^{\circ}$ and $44^{\circ}-54^{\circ}$. Within each band a 1000 point median smoothing of consecutive measurements in each window was applied to the $\mathrm{Mg}$ and $\mathrm{Mg}^{+}$measured column densities. 1000 points corresponds to approximately $.05^{\circ}$ of solar longitude (or $3^{\prime \prime}$ of solar longitude).

Figure 4 shows the $\mathrm{Mg}$ and $\mathrm{Mg}^{+}$column densities in the latitude bands selected for the Geminids and Quadrantids. Also shown in green for reference is the total modeled mass flux rate from Fig. 3, along with vertical black lines as an aid to the eye in marking the peak influx of the two showers and 2 other minor ones. Local maxima in the mass flux occur on either side of the Geminids peak, due to the December Phoenicids and Ursids. The Puppids/Velids, Monocerotids, and $\sigma$ Hydrids showers also occur in the time frame depicted in the plot, but have mass flux rates at least an order of magnitude less than the Geminids and Quadrantids and therefore do not produce a notable peak in the theoretical mass flux rate.

\section{Discussion}

There does not appear to be any correlation between the estimated mass flux rates and enhancements in observed magnesium neutral and ion column densities. In regions where the mass flux rate is nearly constant, i.e. when deposition due to meteor showers is expected to be small, the magnesium column densities oscillate with similar amplitude as during the showers. At times when the mass flux rate is anticipated to increase markedly due to shower activity, no enhancement can be identified in the magnesium column densities. A modeling study by McNeil et al. (2001) predicated an increase in $\mathrm{Mg}^{+}$densities during a meteor showers of at least a factor of 2 (and a factor of 60 increase during a once-a-century meteor "storm"). GOME observations presented here show no indications of changes of that magnitude.

A correlation estimate was made by binning the column densities by solar longitude. All points are grouped into bins of $6^{\prime \prime}$ of solar longitude (one tenth of a degree of solar longitude) and the median value is taken. As an example, this binning results in a linear Pearson correlation coefficient between the $\mathrm{Mg}$ column density and the $\mathrm{Mg}^{+}$column density of 0.48 in the Geminids latitude band $\left(28^{\circ}-38^{\circ}\right.$, Fig. 4) and 0.34 in the Quadrantids latitude band $\left(44^{\circ}-54^{\circ}\right)$. Using bins with a width of $1^{\circ}$ of solar longitude results in correlation coefficients of 0.78 and 0.59 between $\mathrm{Mg}$ and $\mathrm{Mg}^{+}$in the Geminids and Quadrantids bands, respectively. These correlation values are suggestive of a moderate to strong correlation between the neutral and ionized column densities which is not surprising.

Table 3 shows the calculated correlation coefficient of the individual column densities with the estimated mass flux rate variations in the Geminids and Quandrantids latitude bands. These correlation coefficients demonstrate that there is little to no correlation between the increased mass flux during a 
Table 3. Correlation between observed column densities and calculated mass flux rate.

\begin{tabular}{lrr}
\hline Bin Size, degrees of Solar Longitude \& Species & $.1^{\circ}$ & \multicolumn{1}{c}{$1^{\circ}$} \\
\hline Geminids $\mathrm{Mg}$ & .043 & .14 \\
Geminids $\mathrm{Mg}^{+}$ & .015 & .2 \\
Quadrantids $\mathrm{Mg}$ & .066 & -.12 \\
Quadrantids $\mathrm{Mg}^{+}$ & .019 & -.0058 \\
\hline
\end{tabular}

meteor shower and the metal column densities. This supports the qualitative observation of little to no apparent correlation between mass flux rate and column densities.

At the peak of the Geminids shower the estimated mass flux rate is 6 times greater than the daily background value, but the duration of this increase is rather brief. In the time frame covered in Fig. 4 the calculated total mass deposited is $\sim 60 \%$ greater than what would be expected from the background mass flux alone. This averages to about $1.2 \%$ more mass per day due to meteor showers. Given that the lifetime of atomic metals in the MLT is about 6-10 days (Plane, 2004), a perturbation of this magnitude is not likely to produce a large increase in the column density and the deposited metals are simply averaged in with the existing metal layer deposited during the previous 6-10 days. Furthermore, there appears to be no visual evidence of a build-up of metal atoms over the course of a shower. The amount of material ablated in the atmosphere by a shower appears not to be an important source of mass deposition in comparison to the average daily mass flux.

Another factor, which is not central to this analysis, is the annual variation of the sporadic background mass flux rates. Radar reflections from ionized meteor trails and radio reflections have found an annual variation in the number of meteors. The scaled number fluxes of Campbell-Brown and Jones (2006) demonstrates some variability over the course of a year, but the detailed analysis shown in Fig. 4 covers a time span of about 50 days and should not be significantly altered by any annual variation of the mass flux rate.

One factor that may affect the mass flux rate estimate is the different mass ranges which predominate in producing visible meteors (upon which the work of Jenniskens, 1994, is based) versus injecting metal atoms into the atmosphere via ablation. A meteor of mass $\sim 1$ gram is needed to produce a zeroth magnitude meteor and so visual observations from which shower mass indexes and distributions are deduced tend to sample the upper range of ablating meteor masses. Larger mass particles do not contribute significantly to the deposition of metal atoms, due to the relatively low frequency with which they pass through the atmosphere. GOME observations of metal column densities suggest that the mass flux rate does not significantly increase during a meteor shower. The net mass flux rate into the
Earth's atmosphere, and therefore the metal column density, is determined mostly by influx of small particles. The fact that the metal column densities do not increase during meteor showers leads to the conclusion that the mass flux rate of small particles does not increase markedly during a shower, while the larger mass particles causing visible meteors must.

Additionally, even if there is an increase flux of particles across the entire mass scale during a shower, the time of the peak of the mass flux may not coincide with the peak of visual meteors due to phenomena that affect only smaller particles. Smaller masses in a stream are affected by two competing forces: radiation pressure and Poynting-Robertson drag. Radiation pressure tends to move small meteors away from the Sun, while P-R drag causes a loss of momentum and would tend to cause small meteors to spiral inward to the sun. Depending on the mass of the meteor and the geometry of the Earth's path through the stream, the peak flux of smaller meteor masses may occur before or after the visual peak. However, the total mass intercepted by the Earth should not change appreciably.

\section{Conclusions}

There appears to be no obvious correlation between the calculated mass flux rates and observed metal column densities. Correlation between observed GOME Mg and $\mathrm{Mg}^{+}$column densities and calculated mass flux rate in the Geminids and Quadrantids latitude bands in $1^{\circ}$ solar longitude bins is small, $<.2$ for the correlation coefficient. We conclude that meteor showers do not contribute enough mass to cause large scale, long term enhancements of metal column densities. However, the GOME data does not allow us to rule out short term, small scale, local enhancements.

Differences in the mass regimes dominating the average background mass flux and shower mass flux may explain the lack of a shower related. The theoretical mass flux rates is extrapolated from visible meteor rates, which are primarily meteors on the higher side of the mass range. On the other hand, total mass deposition in the atmosphere is dominated by meteor on the lower side of the mass range.

Acknowledgements. We are grateful for the financial support from the United States Air Force, grant number 0710035, and NASA, grant number G06GC53A. We would also like to acknowledge the assistance of W. Dean Pesnell in development of early versions of the GOME data analysis algorithm and Fred Bruhweiler for his suggestions which helped improve this paper.

Edited by: D. J. Cziczo 


\section{References}

Aikin, A. C., Grebowsky, J. M., Burrows, J. P., Correira, J., and Pesnell, W. D.: Temporal evolution of the vertical content of metallic ion and neutral species, J. Atmos. Sol.-Terr. Phs., 67, 1238-1244, doi:10.1016/j.jastp.2005.06.012, 2005.

Campbell-Brown, M. D. and Jones, J.: Annual variation of sporadic radar meteor rates, Mon. Not. R. Astron. Soc., 367, 709-716, doi:10.1111/j.1365-2966.2005.09974.x, 2006.

Ceplecha, Z., Borovička, J., Elford, W. G., Revelle, D. O., Hawkes, R. L., Porubčan, V., and Šimek, M.: Meteor Phenomena and Bodies, Space Sci. Rev., 84, 327-471, doi:10.1023/A: $1005069928850,1998$.

Chandrasekhar, S.: Radiative Transfer, Dover Publications, New York, 1960.

Correira, J., Aikin, A. C., Grebowsky, J. M., Pesnell, W. D., and Burrows, J. P.: Seasonal variations of magnesium atoms in the mesosphere-thermosphere, Geophys. Res. Lett., 35, 6103, doi: 10.1029/2007GL033047, 2008.

Grün, E., Gustafson, B. A. S., Dermott, S., and Fechtig, H.: Interplanetary Dust, edited by: Grün, E., Gustafson, B. A. S., Dermott, S., and Fechtig, H., Astronomy and Astrophysics Library, 2001, ISBN 3-540-42067-3. Berlin, Springer, 804 p., 2001.

Hawkes, L. R.: Television meteors (Invited), in: Meteoroids and their Parent Bodies, edited by: Stohl, J. and Williams, I. P., 227, 1993.

Hawkes, R. L. and Jones, J.: Electro-optical meteor observation techniques and results, Q. J. Roy. Astron. Soc., 27, 569-589, 1986.

Hughes, D.: Meteors and cosmic dust, Endeavour, 21, 31-35, 1997.

Hughes, D. W. and McBride, N.: The mass of meteoroid streams, Mon. Not. R. Astron. Soc., 240, 73-79, 1989.
Jenniskens, P.: Meteor stream activity, I. The annual streams, Astron. Astrophys., 287, 990-1013, 1994.

McNeil, W. J., Dressler, R. A., and Murad, E.: Impact of a major meteor storm on Earth's ionosphere: A modeling study, J. Geophys. Res., 106, 10447-10466, doi:10.1029/2000JA000381, 2001.

Molau, S. and Gural, P. S.: A review of video meteor detection and analysis software, WGN Journal of the International Meteor Organization, 33, 15-20, 2005.

Paxton, L. J. and Anderson, D. E.: Far ultraviolet remote sensing of Venus and Mars, in: Venus and Mars: atmospheres, ionospheres, and solar wind interactions, 113-189 pp., 1992.

Pesnell, W. D. and Grebowsky, J.: Meteoric magnesium ions in the Martian atmosphere, J. Geophys. Res., 105, 1695-1708, doi: 10.1029/1999JE001115, 2000.

Peterson, G.: Dynamics of Meteor Outbursts and Satellite Mitigation Strategies, American Institute of Aeronautics and Astronautics, 1999.

Plane, J. M. C.: A time-resolved model of the mesospheric Na layer: constraints on the meteor input function, Atmos. Chem. Phys., 4, 627-638, 2004, http://www.atmos-chem-phys.net/4/627/2004/.

Rozanov, A., Rozanov, V., Buchwitz, M., Kokhanovsky, A., and Burrows, J. P.: SCIATRAN 2.0 - A new radiative transfer model for geophysical applications in the $1752400 \mathrm{~nm}$ spectral region, Adv. Space Res., 36, 1015-1019, doi:10.1016/j.asr.2005.03.012, 2005.

Williams, I. P.: The Dynamics of Meteoroid streams, Celest. Mech. Dyn. Astr., 81, 103-113, 2001.

Zhou, Q. H., Mathews, J. D., and Zhou, Q. N.: Incoherent scatter radar study of the impact of the meteoric influx on nocturnal Eregion ionization, Geophys. Res. Lett., 26, 1833-1836, doi:10. 1029/1999GL900369, 1999. 